\title{
Fiestas transfronterizas y representaciones espaciales en la frontera México-Texas
}

\section{Cross-border festivals and spatial representations at the Mexico-Texas border}

\author{
Xavier Oliveras-GonZÁlez*
}

\begin{abstract}
Festivals with a cross-border dimension are celebrated in some border communities in which two opposed spatial representations are (re)produced: one of cross-border unity and the other of border separation. The case of Rio Bravo/Grande Region, on the Mexico-USA border, is analyzed; there some sister cities festivals are held. Among the programmed activities there is the so-called hug ceremony, a diplomatic event performed on the international bridges. The present legal, material and psychological enforcement of the border have adversely affected both festivals and spatial representations.
\end{abstract}

Keywords: cross-border festival, spatial representation, sister cities, USA-Mexico border.

\section{Resumen}

En las localidades fronterizas se celebran eventos festivos de carácter transfronterizo donde se (re)producen dos representaciones espaciales opuestas, una de unidad transfronteriza y otra de separación fronteriza. En este trabajo se analiza el caso de la región del río Bravo/Grande en la frontera México-Estados Unidos, donde se celebran las "fiestas de las ciudades hermanas". Entre las actividades programadas se incluye la "ceremonia del abrazo", un acto diplomático realizado en los puentes internacionales. El actual endurecimiento legal, material y psicológico de la frontera ha afectado directamente a las fiestas, así como a las representaciones espaciales.

Palabras clave: fiesta transfronteriza, representación espacial, ciudades hermanas, frontera México-Estados Unidos.

*El Colegio de la Frontera Norte, correo-e: xoliveras@colef.mx 


\section{Introducción}

Los eventos festivos constituyen, como indica Mathisen (2009), un área de estudio complicada puesto que integra diversos elementos: culturales, sociales, políticos, espaciales, económicos, entre otros; asimismo, están abiertos a una multiplicidad de interpretaciones e identificaciones, por lo que, aún cuando antes fueron objeto de estudio casi exclusivo de la antropología, la sociología y el arte, actualmente otras disciplinas se han aproximado al tema. Tal es el caso de la geografía a partir del denominado giro cultural (cultural turn) (Philo, 1999), que supuso "el redescubrimiento de la dimensión cultural en la geografía y su presencia, de una forma u otra, en casi todos los campos de la disciplina" (Lindón y Hiernaux, 2006: 9).

En los años ochenta, sobre todo desde la geografía cultural y del turismo, se empezó a abordar la relación entre espacio y eventos festivos (Janiskee, 1996; De Bres y Davis, 2001; Quinn, 2003, 2005; Getz, 2010), los cuales constituyen una de las muchas prácticas espaciales a través de las que las comunidades construyen y representan sus espacios e identidades territoriales; el interés hacia este campo es creciente y poco a poco se debaten y profundizan varios aspectos que lo conforman.

En esta dirección, el presente trabajo quiere contribuir con el caso particular de los procesos de construcción y representación espacial en las regiones fronterizas y transfronterizas. Un ejercicio previo, aunque con un menor alcance, se realizó para la frontera España-Francia (Oliveras, 2013), caracterizada, como otras fronteras internas de la Unión Europea, por la libertad de movimientos (de personas, capitales y mercancías) y niveles socioeconómicos y socioculturales similares a ambos lados.

Con la intención de contrastar aquellos procesos en otros contextos, la mirada fue dirigida a una hard border, para ello se toma el ejemplo de los eventos festivos que, con carácter transfronterizo, se celebran en la frontera entre México y Estados Unidos de América (EUA). En concreto se analiza el sector equivalente al límite entre Tamaulipas (TS), Nuevo León (NL), Coahuila (CO), Chihuahua $(\mathrm{CH})$ y Texas (TX), que coincide con el curso del río Bravo/Grande (topónimos usados en cada uno de los dos países).

Aquí se han desarrollado varios pares de localidades geográficamente contiguas situadas una frente a la otra, las denominadas ciudades hermanas o gemelas, twin cities o sister cities $^{1}$ (Arreola y Curtis, 1993; Kearney

\footnotetext{
${ }^{1}$ No debe confundirse con la suscripción de un acuerdo de hermanamiento, mediante el cual localidades - no geográficamente contiguas- se convierten en hermanas. Ello no excluye que algunas ciudades de esta frontera se hayan también hermanado, como Camargo (TS) y Río Grande City (TX), Matamoros (TS) y Brownsville (TX) o Nuevo Laredo (TS) y Laredo (TX).
} 
y Knopp, 1995), cuyas interrelaciones están condicionadas por el curso fluvial y la frontera (figura 1), así como por los puentes internacionales que permiten cruzar el río/frontera y unir las localidades. Se puede decir que estas infraestructuras constituyen los ejes fundamentales de una articulación regional transfronteriza, a la par que, en las fiestas, se hace un uso material y simbólico de ellos.

Constituyen además uno de los símbolos universales de la amistad, la buena vecindad y la voluntad de superar los efectos adversos de la frontera. Las fiestas allí celebradas se conocen genéricamente como "fiestas de las ciudades hermanas", por llevarse a cabo en aquellos pares, o "fiestas de la buena vecindad", por ser uno de los dos motivos principales de celebración, junto al turismo.

La primera de la que se tiene constancia histórica es la celebrada en Laredo (TX), desde 1898 (Green, 1992; Klein, 1997). La elección de este espacio geográfico viene a suplir también su ausencia en los estudios geoculturales de la región fronteriza México-euA; sirvan de ejemplo los trabajos de Daniel D. Arreola, reconocido por sus aportaciones (1993, 2013), donde sólo hay menciones esporádicas.

Las particularidades de las fiestas y de otras prácticas espaciales, así como de las representaciones, responden a los contextos históricos de la frontera donde se ubican y se desarrollan. En este sentido, celebran y son, a la vez, resultado de la interrelación económica, política, social y cultural entre ambos lados.

Como se mostrará, las fiestas transfronterizas han incorporado la representación de la región fronteriza México-EuA como una singularidad, que deriva de lo que Dear (2013) identifica como las integraciones materiales y psicogeográficas.

En el plano material se incluyen procesos como una alta integración económica, una densa movilidad transfronteriza y la planificación y realización compartida de proyectos de desarrollo regional; en el psicogeográfico, la influencia e hibridación cultural y la formación de imaginarios e identidades transfronterizas y binacionales.

En los ámbitos académico y periodístico de los años ochenta, dichas integraciones llevaron a identificar aquella región transfronteriza como un "tercer país" o una "tercera nación" (Anzaldúa, 2007; Dear, 2013); es decir, como un espacio local y regional intermedio e híbrido geográfica y culturalmente entre los dos estados-nación.

En esta identificación resuena una línea conceptual semejante al "tercer espacio" desarrollado por Hommi Bhabha (1990) y por Edward Soja (1996). Otras investigaciones, por el contrario, relativizan aquellos procesos y rebaten tales interpretaciones, enfatizando los contrastes, desigualdades y violencias, así como los miedos a la americanización en 


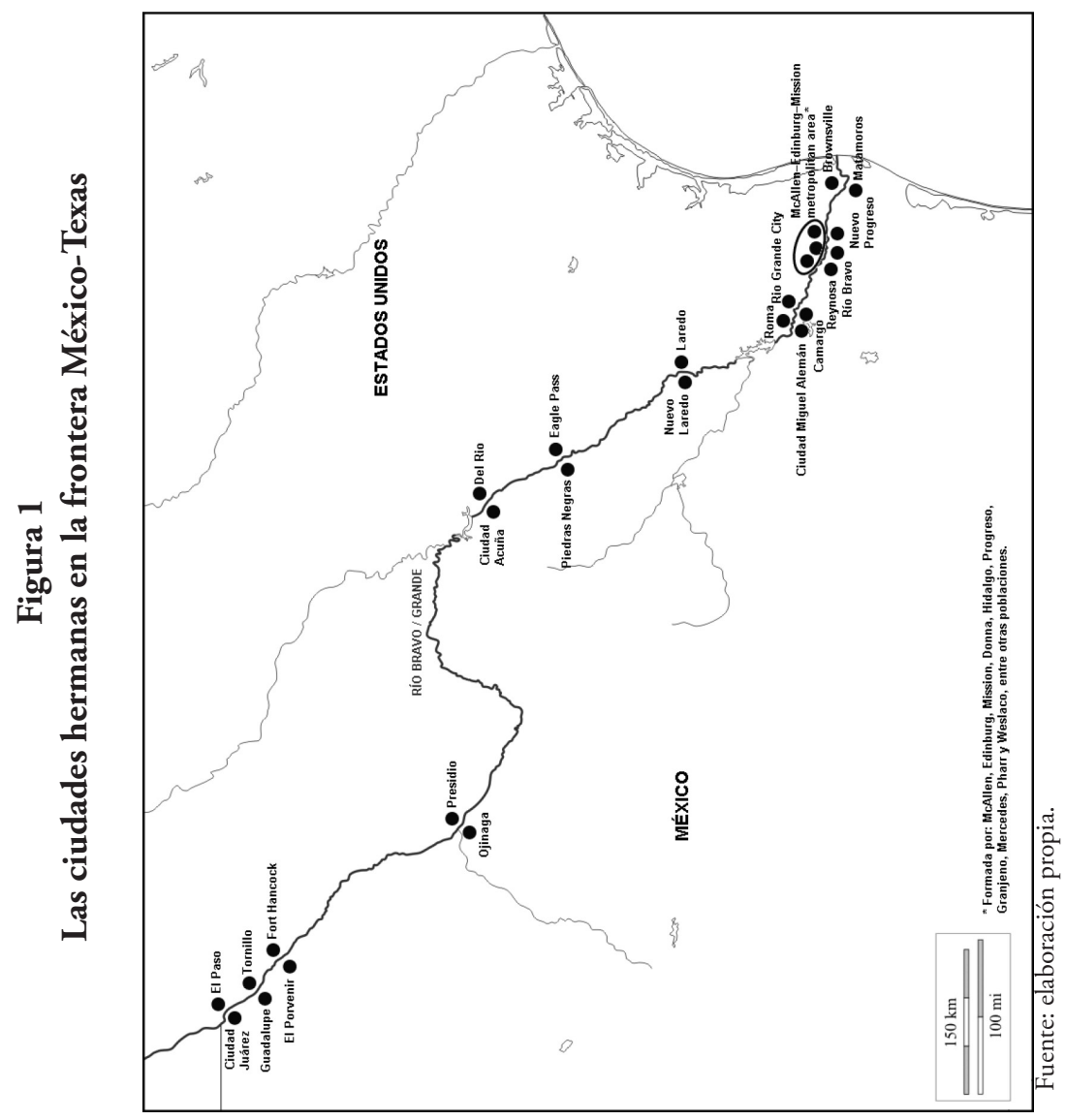


México y a la mexicanización en euA (Arreola y Curtis, 1993; Pastor y Castañeda, 1988).

Sin perder de vista ese debate, el estudio presta especial atención a las fiestas en el actual contexto fronterizo, caracterizado por un notable endurecimiento legal, material y psicológico dado a partir de 2006 (política fronteriza y migratoria de EUA más restrictiva y aumento de la inseguridad asociada al narcotráfico y al crimen organizado en México) que podría estar causando una ruptura de las interrelaciones y, con ello, de las fiestas.

Del conjunto de actos incluidos en sus programas, es en la ceremonia del abrazo donde se manifiestan más claramente las representaciones espaciales. Este acto se celebra en medio de los puentes internacionales, justo en la guardarraya, donde se encuentran las delegaciones de ambos lados, integradas por los alcaldes y otras autoridades (básicamente políticas y económicas de nivel local, pero también de nivel estatal y federal).

Como se puede intuir, este grupo social (y las relaciones de poder que lo atraviesan) incorpora un sesgo sociopolítico particular a la generación de los discursos y a la apropiación del espacio, que condiciona las representaciones espaciales. Aunque no sea el objetivo del presente artículo, se puede apuntar que sus representaciones difieren de las producidas por otros grupos sociales como las clases populares mexicana, mexicanoamericana y angloamericana analizadas por Richardson (1999) y Vila (2000), y los niños y niñas de ambos lados, por Zúñiga (1998). De ello se extrae que no existe una homogeneidad en las representaciones espaciales de la frontera México-EuA.

Esta investigación responde a dos preguntas básicas: cuáles son las representaciones espaciales que se (re)producen en la ceremonia del abrazo y cuáles son los efectos del endurecimiento fronterizo en las fiestas y en las representaciones. Se analizan los componentes discursivos (re)producidos por las autoridades participantes y los lugares (la frontera/el río/los puentes) donde éstos se (re)producen y que dichas autoridades se apropian.

De acuerdo a los objetivos y al arco temporal de estudio se emplearon distintas fuentes: primero se procedió a la revisión de notas de prensa, reseñas en los medios de comunicación y documentación escrita y gráfica (fotografías) de las fiestas celebradas en el período 2006-2013; lo mismo para las entidades organizadoras.

En segundo lugar se llevó a cabo la observación directa de algunas fiestas celebradas en 2013 (Matamoros-Brownsville, Nuevo ProgresoProgreso y Reynosa-Hidalgo), y se realizaron -cuando fue posibleentrevistas y valoraciones cualitativas con miembros de las entidades organizadoras. Finalmente, se revisaron estudios sobre las fiestas (ver fuentes consultadas). 
Para la exposición se discute, en primer término, el concepto de fiesta transfronteriza y las categorías de representación espacial en el espacio transfronterizo; en segundo se describen las fiestas de las ciudades hermanas, con especial atención a la ceremonia del abrazo; en tercer término se consideran los elementos contextuales que permitan la comprensión de su evolución. Por último, se muestran las representaciones identificadas en aquella ceremonia en el actual contexto fronterizo.

\section{Las fiestas transfronterizas como práctica y espacio de representaciones espaciales}

Como se apuntó en la introducción, el espacio de las fiestas transfronterizas y las representaciones espaciales que en ellas se (re)producen ha sido analizado desde la geografía de manera creciente. Ahora bien, la mayoría de aproximaciones se centran en estudios de caso o se abordan parcialmente en investigaciones sobre turismo y cultura en espacios fronterizos; no existen análisis teóricos y empíricos de conjunto.

En el cuadro 1 se citan algunos ejemplos de estudios de caso a los que se pueden sumar los mencionados en el apartado tres en relación a la frontera México-eua. Con base en ellos se esboza una aproximación teórica relativa a las fiestas transfronterizas y a las representaciones espaciales. Desde una perspectiva geográfica los eventos festivos son abordados como una práctica espacial; es decir, como un modo por el cual los agentes sociales se apropian, moldean y producen -material y simbólicamente- un espacio, en este caso por medio de la organización y celebración de las fiestas.

Mediante dicha práctica, localizada en un espacio concreto, las comunidades locales se conectan con su espacio, y elaboran, codifican y otorgan un conjunto de representaciones espaciales, esto es, los significados y valores sobre el lugar de celebración, los espacios fronterizos (el propio y el del otro lado) y transfronterizos, sus elementos e interrelaciones (Quinn, 2003).

En primer lugar, tomando como referencia la definición amplia que Janiskee (1996) ofrece para el concepto de fiesta (festival, en el original en inglés), la transfronteriza es aquel "evento festivo formal, colectivo y periódico que reúne a las comunidades locales de ambos lados de una frontera para celebrar algún objeto, suceso o hecho".

Como en el debate sobre el concepto de tercer país apuntado en la introducción, en las fiestas también se encuentra la misma doble interpretación, por una parte -como se deduce de Diamond (2012) al hablar de las fronteras en las sociedades tradicionales- estos eventos tienen una función ritual y sociopolítica de mantenimiento de paz y cordialidad entre las comunidades fronterizas, de reafirmación de compromisos, 
asimismo son una manera de evitar posibles conflictos derivados de la vecindad fronteriza.

Por otra parte, como se deduce de García Canclini (2003) y Grimson (2004), la función de las fiestas transfronterizas está sujeta a otra posible interpretación, en tanto que se representan en una aparente e ingenua coexistencia fronteriza e integración supranacional. En este sentido, su función consiste en minimizar e, incluso, ocultar las asimetrías de poder entre un lado y otro de la frontera, la homogeneización desigual y los conflictos.

Siguiendo a Brunner (1992), se trata de construir una representación en absoluto incómoda y completamente asumible para quien ejerce mayor poder en dicha relación asimétrica. En definitiva, las fiestas serían una reproducción y una perpetuación de las relaciones de poder. Ambas interpretaciones señaladas no son excluyentes - por el contrario- pueden darse simultáneamente.

Para efectos conceptuales y metodológicos es necesario precisar que la formalidad de la fiesta deriva de su institucionalización y legitimación por parte de las autoridades locales (políticas, económicas, sociales o religiosas) y cuenta con una organización y planificación previa a su celebración; su carácter colectivo procede del hecho que representa el conjunto de la comunidad local y una parte asume (y presume) la representación del conjunto, adicionalmente, su periodicidad es, generalmente, anual.

Gracias a su carácter formal y colectivo se acentúan y evidencian más claramente las representaciones espaciales. En lo que respecta a los motivos de festejo, se detecta una amplia variedad que incluye la tradición cultural y religiosa, la conmemoración de hechos históricos, la exhibición artística (cine, teatro, música, danza, etc.), la exhibición de productos agrarios e industriales, la promoción turística, el hermanamiento, amistad y buena vecindad y la reivindicación regional, entre otros.

Más que los motivos particulares de celebración, lo distintivo de los eventos transfronterizos es la interacción con y entre las poblaciones de ambos lados de una línea fronteriza en y durante el espacio-tiempo que duran. Lo relevante es la mediatización de las comunidades por la frontera o, dicho de otra forma, la intervención de aquélla en los procesos de construcción que las poblaciones hacen de sí mismas, de su lado y del otro, y su sentido, así como la transformación de las relaciones sociales y entre las instituciones y organizaciones de ambos lados.

La atención en este caso se centra en los espacios y las identidades territoriales (fronteriza, transfronteriza, nacional, binacional) que se celebran y producen. Esta vinculación entre comunidad, espacio y frontera se manifiesta en la totalidad del evento o, en ocasiones, únicamente en algunas de las actividades que conforman el programa. 


\section{Cuadro 1 \\ Casos de estudio sobre fiestas transfronterizas}

\begin{tabular}{lll}
\hline \multicolumn{1}{c}{ Frontera } & \multicolumn{1}{c}{ Caso de estudio } & \multicolumn{1}{c}{ Referencia } \\
\hline Canadá-EuA & $\begin{array}{l}\text { International Friendship Festival (Fort Erie } \\
\text { y Buffalo) }\end{array}$ & Eagles, 2010 \\
México-Eua & $\begin{array}{l}\text { Fiesta del Río (Imperial Beach y Tijuana) } \\
\text { Festival Internacional de la Raza }\end{array}$ & Conway, 2010 \\
& Bustamente, 2012 \\
Colombia-Perú-Brasil & Festival de la confraternidad amazónica & Unigarro, 2012 \\
Albania-Grecia & Romerías & De Rapper, 2010 \\
R.Checa-Eslovaquia & Festival de la Euroregión Carpatia Blanca & Carmin et al., 2003 \\
Alemania-Austria- & Festivales teatrales (lago Constanza) & Wilkinson, 2007 \\
Suiza & & \\
Suecia-Finlandia & Fiesta del Poikkinainti (bodas transfronte- & Prokkola, 2010 \\
rizas) & Finnskogdagene (Día del bosque Finn) & Mathisen, 2009 \\
Suecia-Noruega & Día de la Cerdańa & Oliveras, 2013 \\
España-Francia & Tributo de las Tres Vacas & Fernández de Casa- \\
& & devante, 1989 \\
\hline
\end{tabular}

Fuente: elaboración propia.

Las representaciones espaciales se refieren al espacio transfronterizo (un espacio transversal a una frontera) y a los espacios fronterizos (espacios geográficamente contiguos divididos por una frontera). Ambos conceptos y su relación con la producción de representaciones has sido abordados desde los llamados border studies; especialmente desde la aproximación teórica de la construcción de regiones transfronterizas (Paasi 1996; Perkmann 2003; Hinfray 2007; Trillo y Lois 2011; Breugnot 2012). Las representaciones están constituidas por los rasgos, significados y valores geográficos, culturales, sociales, económicos o históricos atribuidos al espacio.

Del conjunto de componentes que las constituyen cabe destacar la frontera, en todas sus posibles formas -física, jurídica, política, social o cultural- puesto que interviene en su proceso de producción, las estructura y les da sentido (Hamman, 2003; Migdal, 2004; Wilkinson, 2007; Benedetti, 2007; Conway, 2010).

En estos espacios confluyen e interactúan las prácticas de las comunidades de ambos lados y las políticas fronterizas de los estados-nación (que, simplificando, abarcan desde la integración transnacional, en un extremo, al control fronterizo, en el otro). Como resultado, los espacios fluyen simultáneamente en dos ejes: uno, de oposición entre lo local y lo nacio- 
nal, y otro, de oposición entre la integración y la fortificación. Desde ambos ejes se les dota de contenido integrando elementos locales y nacionales (de cada uno de los estados-nación) y de atracción y repulsión. Esta dialéctica entre lo local y lo nacional, entre la integración y la fortificación, también confluye y se refleja en las fiestas transfronterizas y en las representaciones espaciales.

Resulta posible distinguir dos categorías básicas aparentemente opuestas: una, el conjunto de representaciones que Hamman (2003) denomina de unidad transfronteriza y otra, que aquí identificamos como de separación fronteriza (figura 2); esta segunda, rara vez es analizada en la literatura académica sobre espacios transfronterizos, puesto que las investigaciones se concentran en las dinámicas que permiten explicar la construcción de estos espacios. Sin embargo, como Grimson (2000, 2004) sugiere, sólo la combinación de ambas permite un análisis fecundo y crítico acerca de las fronteras y las prácticas espaciales.

\section{Figura 2 \\ Representaciones espaciales en espacios fronterizos geográficamente contiguos}

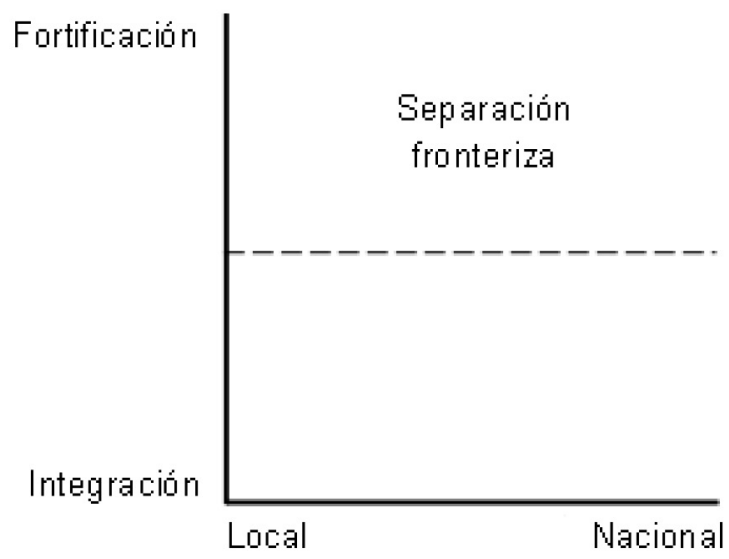

Fuente: elaboración propia.

Por lo general, en las representaciones de unidad transfronteriza prevalece el interés local, mientras que en las de separación fronteriza, el nivel y el interés nacional, que se traslada a la escala local, llega incluso a suplantarlo. En las primeras, la geografía, el tiempo histórico o las dinámicas sociales, culturales y económicas se utilizan como recursos particulares para argumentar la integración del espacio transfronterizo, el reconocimiento de la singularidad de este espacio y de su diferencia respecto a los espacios e identidades de los estados-nación. 
Así, por ejemplo, se construyen ideas de proximidad geográfica, de conformidad histórica respecto a los territorios pasados, presentes y proyectados, y de dinámicas complementarias e interdependientes. En el espectro opuesto, en las segundas, se utiliza la geografía, la historia, entre otras, para reafirmar la frontera como división, la soberanía de los estadosnación y las adscripciones e identidades nacionales. En este caso la proximidad geográfica se percibe como una amenaza, el tiempo histórico se (re)interpreta para construir discontinuidades y se refuerzan las diferencias sociales, culturales y económicas.

La formación de estas representaciones es un proceso dinámico y variable, en tanto que cambian en el tiempo y en el espacio y en función de contingencias coyunturales; lo pueden hacer de un individuo y grupo social a otro de acuerdo a sus experiencias particulares con el estado-nación, la frontera y el otro lado.

Ello se refleja en una basculación de las representaciones entre las dos categorías mencionadas que aparecen en grados variables entre unas y otras. Sirviéndonos de los ejemplos citados en el cuadro 1, se pueden situar festividades como las celebradas en la triple frontera ColombiaPerú-Brasil, donde se festeja la pertenencia nacional a cada uno de los tres países, pero en las que también hay espacio-tiempos (pocos) para la hermandad (como un abrazo entre las reinas de las fiestas) (Unigarro, 2012).

En el extremo opuesto se hallan casos como el del Día de la Cerdaña, en la frontera España-Francia, donde en los discursos oficiales se insiste en la inexistencia de la frontera y se refuerza la unidad transfronteriza (inclusive con una bandera y un himno propios); además se ha convertido en un espacio-tiempo de encuentro de los alcaldes de ambos lados para debatir y planificar proyectos de cooperación transfronteriza. A pesar de ello, la fiesta se celebra alternativamente en una localidad de un lado y otro de la frontera, reconociendo implícitamente su existencia (Oliveras, 2013).

\section{Espacio-tiempos transfronterizos de las fiestas de las ciudades hermanas}

Las fiestas transfronterizas en la frontera del río Bravo/Grande comparten tres características: la primera es la voluntad expresada de incluir a las comunidades de ambos lados de la frontera, tanto si la organización recae en una de ellas o en ambas, o si las entidades organizadoras son gobiernos locales, cámaras de comercio o asociaciones civiles; la segunda incluye distintas actividades (muestras de cultura popular, conciertos, exposiciones, desfiles, competiciones deportivas, bailes, concursos), que pueden diferir unas de otras pero que cuentan con al menos un acto de reafirmación de amistad y buena vecindad fronteriza. 
La más representativa de ellas es la ceremonia del abrazo (en ocasiones también llamada del saludo binacional, del puente internacional o hands across the border). A ésta se suman, si bien no es común a todas, otros actos con el mismo objetivo, tales como los desfiles binacionales y carreras pedestres y ciclistas.

En todos ellos los puentes internacionales constituyen un elemento central, tanto a nivel material como simbólico; la ceremonia del abrazo se celebra en ellos, mientras que los itinerarios de los desfiles y actividades deportivas los cruzan puesto que se inician en una de las ciudades y finalizan en la otra. Asimismo, participan individuos y grupos de ambos lados de la frontera.

La tercera característica, junto a la motivación de la amistad y la buena vecindad, se añade la promoción turística y económica de los espacios fronterizos y transfronterizos.

Al tomar como indicador la inclusión de la ceremonia del abrazo, en el área de estudio se han reportado trece fiestas (figura 3) correspondientes a diez pares de ciudades, la mayoría localizadas en la frontera Tamaulipas-Texas, el sector con mayor número de núcleos urbanos.

De éstas, la fiesta más renombrada es la Washington's Birthday Celebration de Laredo (TX), que ha sido ampliamente estudiada (Green, 1992; Dennis, 1997; Klein, 1997; Young, 1998; Peña, 2006); en menor medida también han sido objeto de investigación las fiestas mexicanas de Matamoros (TS) y la Charro days fiesta de Bronwsville (TX), que se celebran en las mismas fechas (Wooldridge y Vezzetti 1989; Knopp et al. 2009; Dávila 2013).

Se han excluido del análisis otras actividades como la Fiesta de las Flores de El Paso (TX) (Fernández y Venegas 2010), el Sister City International Festival de Laredo (TX), la Texas Citrus Fiesta de Mission (TX) o el Friendship Festival de Granjeno (TX) (Dorsey y Díaz-Barriga 2010).

Por otra parte, la ceremonia del abrazo o eventos similares no es exclusiva de estas fiestas. También en la frontera México-EuA, entre Naco, Sonora, y Naco, Arizona, se lleva a cabo desde 1979 un evento transfronterizo (actualmente denominado Fiesta Bi-Nacional), durante el cual los alcaldes se dan la mano a través del muro fronterizo, reclamando que en vez de estar limitados a un apretón debería ser posible un abrazo.

De forma similar, en el sector del río Bravo/Grande, en 2007 varios pares de ciudades realizaron el Hands across el Río para protestar contra la construcción del muro. Igualmente se han celebrado otros actos de protesta que emulan el abrazo, como el Abrazo ecológico de hermandad de 2012 entre Piedras Negras (CO) y Eagle Pass (TX) para reivindicar la protección medioambiental del río y de los espacios aledaños. El abrazo 


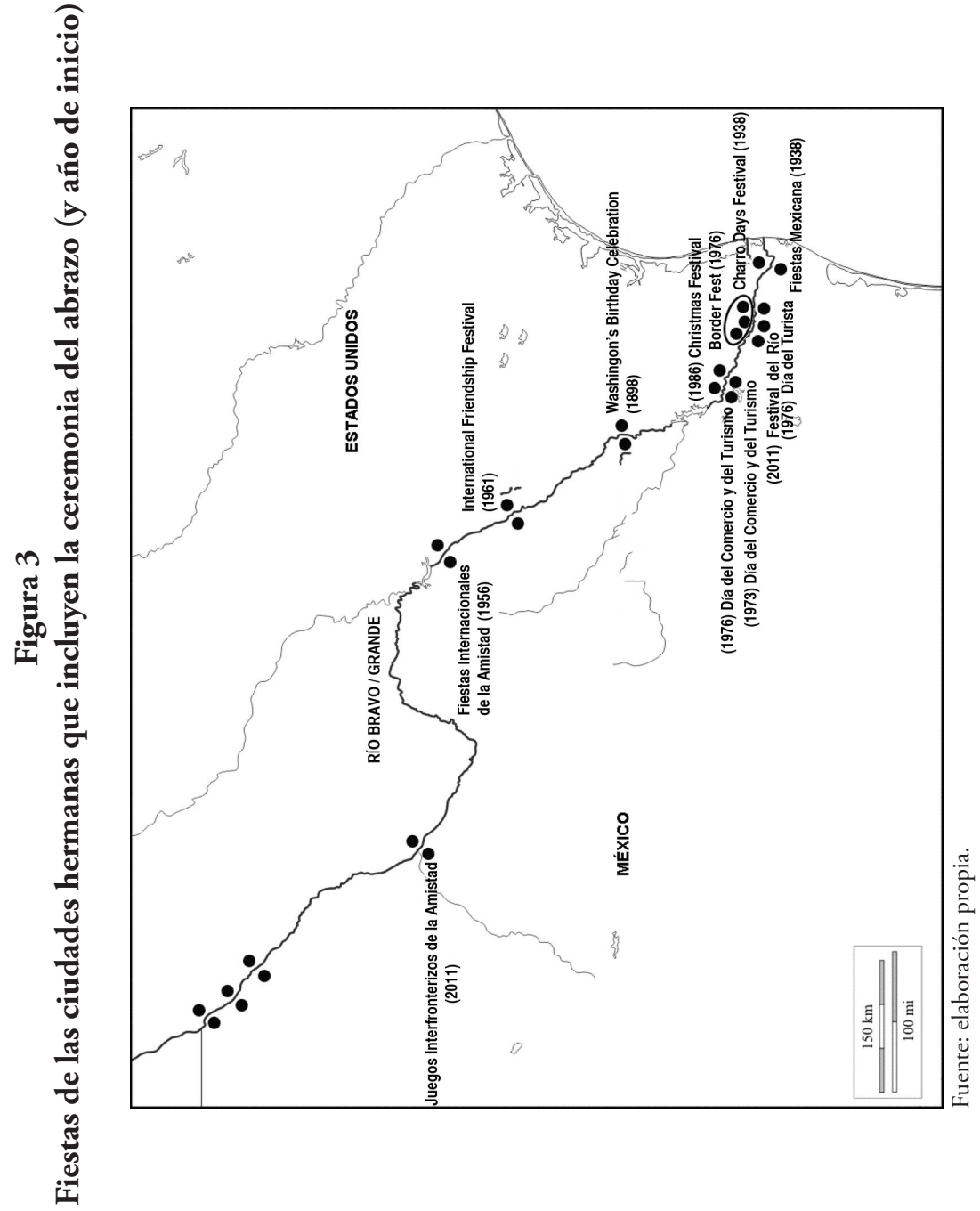


también se ha celebrado en otras fronteras con objetivos parecidos, tal es el evento entre las ciudades de Arica (Chile) y Tacna (Perú). ${ }^{2}$

De acuerdo a la doble interpretación antes expuesta, el objetivo principal de la ceremonia del abrazo puede ser fortalecer la amistad y la buena vecindad entre las comunidades fronterizas y los dos países o, por el contrario, perpetuar la asimetría de poder y las desigualdades existentes entre ambos. Sea cual sea, se trata de un acto estrictamente diplomático, cuyo protocolo varía en función del nivel de las autoridades asistentes. Como consecuencia de ello es remarcable el desinterés del resto de la población por participar, en los casos que es posible, en la ceremonia, así como la exclusión, en otros.

Desde cada extremo del puente, las comitivas de cada uno de los lados de la frontera se acercan a la guardarraya donde se encuentran. Se reúnen las autoridades locales y representantes económicos y civiles y les acompañan autoridades estatales y federales, así como militares y religiosas, que otorgan una mayor legitimidad. El acto incluye un abrazo entre los alcaldes y entre el resto de autoridades de un mismo o equivalente nivel, seguido de sus parlamentos.

En ocasiones la actividad incluye un abrazo infantil entre parejas de niños y niñas de ambas ciudades vestidos con trajes tradicionales, el intercambio de banderas de México y eua y la entonación de los respectivos himnos nacionales. Suele ser también común la presencia de un cargo honorífico cuyo título refleja el objetivo de la fiesta: los Miss y Mister amigo, amistad, friendship e internacional o turista, concedido a personalidades mexicanas o estadounidenses que, a juicio de la entidad organizadora, hayan contribuido al acercamiento entre las dos ciudades o países desde sus respectivos campos (arte, deporte, ciencia, medicina, política, entre otros).

Como ya se ha apuntado, la ceremonia se lleva a cabo en medio de los puentes internacionales, un lugar que no únicamente se refiere al espacio físico donde se ubica, sino que ellos mismos contribuyen a la producción de las representaciones espaciales. La asociación entre la ceremonia y los puentes está sujeta a cuatro consideraciones previas: la primera consideración es que el surgimiento de la ceremonia responde a la previa apertura (o reapertura, en algunos casos) de los puentes. La construcción de puentes en el río Bravo/Grande se inició a fines del siglo XIx (anteriormente el cruce se realizaba por medio de chalán ${ }^{3}$ y ferry), primero rudi-

\footnotetext{
${ }^{2}$ La amistad transfronteriza y binacional se ha celebrado de forma irregular desde 1937, con actos como el Abrazo de la concordia, en 1974; Abrazo del bicentenario, en 2010 o el Abrazo de la paz en 2012.

${ }^{3}$ Actualmente sólo pervive el chalán -embarcación menor de fondo plano para transporte en ríos y lagos de poco calado- entre Díaz Ordaz (TS) y Los Ébanos (TX).
} 
mentarios y de madera, luego adaptados al tráfico vehicular y, en algunos casos, ferroviario (Arreola, 2013).

En este sentido, la ceremonia constituye además una celebración de los avances tecnológicos y urbanísticos. Sirva de ejemplo la ceremonia entre Matamoros y Brownsville, celebrada originalmente en el puente B\&M (construido en 1909), pero que se trasladó al puente Puerta México, inaugurado en 1970, por ser más moderno.

La segunda consideración se refiere a que, en un espacio divido por un curso fluvial, los puentes son el único lugar que permite a las comitivas encontrarse en el límite entre ambos lados, evitando tener que celebrarla en un lado o en otro

La última consideración se da al tener en cuenta el objetivo turístico de las fiestas, en la que los puentes son la primera y una de las principales atracciones turísticas e iconos visuales de las ciudades fronterizas, puesto que son a la vez frontera y puerta de entrada para los visitantes de "el otro lado". Como señala Arreola (2013), esta centralidad la adquieren desde que, durante el primer tercio del siglo $\mathrm{xx}$, se inicia el turismo estadounidense a las ciudades mexicanas del río Bravo.

Como última consideración, los puentes tienen -casi universalmenteuna connotación positiva en términos de acercar las personas, forjar enlaces y reconciliarse; en este sentido, los internacionales posiblemente sean el símbolo por antonomasia de los espacios y la cooperación transfronterizos y de la superación de las divisiones (O’Dowd, 2002; Warnaby y Medway, 2008).

\section{Evolución en un contexto local-nacional y de integración- fortificación cambiante}

Antes de entrar de lleno en la identificación de las representaciones espaciales, todavía es necesario mostrar la evolución de la ceremonia del abrazo y, en general, de las fiestas de acuerdo a un cambiante contexto fronterizo. Además de estar apoyadas en aspectos locales, a los que no se entrará en detalle, su evolución responde a los sucesivos cambios en las dialécticas entre lo local y lo nacional, y entre integración y fortificación en la frontera México-EuA.

Con base en el surgimiento de las fiestas y la evolución de las políticas fronterizas, que aparecen directamente relacionadas, se han identificado a grandes rasgos cuatro periodos: 1900-1950; 1951-1970; 1971-1990 y 1991 en adelante.

Durante la primera mitad del siglo XX se celebran únicamente fiestas en Nuevo Laredo-Laredo y Matamoros-Brownsville. La Washington's Birthday Celebration (wBC), en Laredo (TX), correspondía originalmente 
a un acto de enaltecimiento patriótico donde se exhibía y representaba la superioridad angloamericana sobre la población de origen mexicano e indígena, aprovechando, como su nombre indica, el natalicio de uno de los padres fundadores de EUA.

En la década de 1930 se renovó la fiesta y se inició la Charro Days Fiesta en Brownsville (TX) y las fiestas mexicanas en Matamoros (TS), así como la Texas Citrus Fiesta de Mission (TX), cuyo origen se halla en la promoción comercial y turística de estas localidades.

Tanto éstas como los cambios introducidos en Laredo se implementan en plena depresión económica, amplificada por el fin de la llamada Era de la Prohibición, lo que había significado un primer impulso turístico para las ciudades fronterizas (Arreola, 2013). En este sentido, las fiestas fueron una innovación para impulsar de nuevo el turismo.

Desde la primera edición de la WBC se invitó a participar a Nuevo Laredo (TS) con una recepción de bienvenida en el City Hall. Con los cambios de los años 1930, la recepción se convirtió en un acto oficial en medio del puente (es decir, antes de cruzar la frontera), donde las respectivas autoridades se estrechaban las manos. Lo mismo se hizo en Matamoros-Brownsville, donde se integró la ceremonia del saludo binacional con un apretón de manos en el puente.

Poco después, en el marco de la Segunda Guerra Mundial, la ceremonia adquirió mayor carácter diplomático, puesto que se utilizó para mostrar la alianza entre ambos países, participaban autoridades políticas y militares de nivel estatal y federal. Si bien terminado el conflicto bélico su relevancia diplomática disminuyó, ya no la perdió.

En las décadas de los cincuenta y sesenta, las fiestas precedentes -más las que surgieron: Ciudad Acuña-Del Río y Piedras Negras-Eagle Pass- se inscriben en la etapa de mayor turismo estadounidense en las ciudades fronterizas del río Bravo. Sin embargo, también lo hacen en un contexto de creciente endurecimiento físico y jurídico de la frontera y de hostigamiento de la población mexicana por parte de EUA, quien puso en marcha una estricta política fronteriza y migratoria, desde la Operación Wet Back en 1954 hasta la Operación Intercepción en 1969, pasando por la clausura del Programa bracero en 1964 (se había iniciado en 1942 para permitir la inmigración de mano de obra mexicana).

Ello tuvo un impacto directo en las fiestas, porque alteró su desarrollo: la Operación Intercepción terminó con el "paso libre" (la suspensión del chequeo de documentos) que se aplicaba durante su celebración (Knopp et al. 2009; Dávila 2013). Asimismo, como señalan Fernández y Venegas (2010: 126) para la Fiesta de las flores de El Paso (TX), "parece que las condiciones de hostilidad contra los latinos desempeñaron un importante papel en la instauración de esta tradición festiva”. El objetivo era doble: 
reivindicar la población, la cultura y la identidad mexicoamericana, frente a la angloamericana, haciendo de las fiestas eventos más populares e integradores, y redirigir amistosamente las relaciones México-EUA.

No es casualidad que las nuevas fiestas, e incluso la WBC, se organizaran o coorganizaran por las secciones locales de dos asociaciones promexicanas: la League of United Latin American Citizens (LULAC) y el Consejo Internacional de la Buena Vecindad (CIBv)/International Good Neighbor Council (IGNC). ${ }^{4}$

En este contexto se transformó la ceremonia binacional, sustituyendo el apretón de manos por el abrazo; el cambio se produjo a raíz de un hecho protagonizado por los presidentes de ambos países: durante la firma del acuerdo para la construcción de la presa internacional de la Amistad, ${ }^{5}$ en Ciudad Acuña en 1960, los presidentes Adolfo López Mateos y Dwight D. Eisenhower se abrazaron, gesto que desde entonces se emuló en las fiestas. Con la escenificación del abrazo se equipara el nivel nacional con el local, pero además encierra un mayor simbolismo, por lo que implica de mayor contacto corporal y proximidad. Para la población mexicana y mexicoamericana es un gesto habitual de amistad, familiaridad, respeto y confianza, mientras que la angloamericana está poco habituada a él, más aún entre hombres (Villa, 2000).

En la década de los setenta y ochenta se implantaron cinco festividades: tres en poblaciones mexicanas (Camargo, Miguel Alemán y Nuevo Progreso) y dos en el lado estadounidense (Hidalgo y Rio Grande City). De nuevo surgen con una clara voluntad turística y comercial, como prueba está que la iniciativa fue tomada por las cámaras de comercio (y que la Laredo Chamber of Commerce se sumara a la WBC) así como el nombre de las fiestas del lado mexicano (Día del turista y Día del turismo y del Comercio).

Mediante las fiestas, las ciudades buscan fortalecer la economía regional y el sector turístico, cada vez más dependiente de los llamados winter texans, grupo conformado por migrantes temporales, en su mayoría jubilados, procedentes de las regiones frías de EuA y Canadá que en invierno residen en el sur de Texas, más cálido y más cercano a los servicios y productos médicos -baratos- de México (Dalstrom, 2012). La wвC y la Border fest de Hidalgo (TX) son las fiestas que, en los últimos treinta años, han captado más visitantes, mientras que el Día del turista de Nuevo Progreso (TS) es el que con mayor claridad se dirige a los winter texans.

${ }^{4}$ La LULAC se fundó en 1930 en Corpus Christi (TX), es una de las más importantes en EUA para la defensa de los derechos de la población hispana; el cibv, en 1954 en Monterrey (NL), fomenta las relaciones amistosas entre México y EUA.

${ }^{5}$ El nombre elegido para la presa es también indicativo de esta misma coyuntura, originalmente se le denominó "Sitio del diablo", por el afluente del río Bravo que desemboca allí. 
Las nuevas fiestas incorporaron la ceremonia del abrazo como acto de apertura oficial, dando legitimidad a los eventos turísticos y a su carácter transfronterizo. Aunque el nivel nacional parece no estar tan presente como en el periodo anterior, se continúa manifestando, tal es el origen de la Border fest, organizado por primera vez en 1976 para conmemorar el bicentenario de Estados Unidos, efeméride que también fue celebrada en el resto de fiestas organizadas en el lado estadounidense.

Un último periodo se inicia en la década de los noventa y continúa en el siglo XXI, está determinado por una ampliación de la dicotomía entre integración y fortificación: en primer lugar se da una creciente integración de América del Norte, especialmente en el campo económico con el Tratado de Libre Comercio América del Norte (TLCAN), pero también en el medioambiental, con el Acuerdo de Cooperación Ambiental de América del Norte (ACAAN), ambos en vigor desde 1994.

Si bien se trata de procesos binacionales, ambos tratados actuaron como factores coyunturales para el incremento de la paradiplomacia, es decir, de la acción exterior de los gobiernos locales y estatales. En el caso de las localidades fronterizas se tradujo en la reafirmación de los lazos de buena vecindad, ya fuera mediante las fiestas o con la suscripción de varios acuerdos de hermanamiento y de cooperación transfronteriza.

Paralelamente, sobre todo a partir de los hechos del 11 de septiembre de 2001, se produjo un endurecimiento material, legal y psicológico de la frontera. Esta condición se intensificó a partir de 2006, como resultado de dos procesos paralelos, uno en cada lado.

En EUA se han diseñado y aplicado políticas fronterizas y migratorias más restrictivas, especialmente como consecuencia y pretex to del terrorismo internacional, lo que se ha traducido en un endurecimiento material y legal de la infraestructura y control (protección, aduana e internamiento) y migratorio.

Resulta paradigmática la construcción del muro; si bien se inició en San Diego California en 1994, se desarrolló plenamente con la Secure Fence Act de 2006, cuyas labores en Texas se emprendieron en 2007 con la intención de extender una barda continua entre Brownsville y Laredo.

La violencia e inseguridad en México, asociada con el combate iniciado en 2006 entre las organizaciones criminales vinculadas al narcotráfico, así como entre éstas y el Estado, ha traído consecuencias como la disminución de la movilidad transfronteriza desde EUA, de igual manera se han clausurado (o se han traslado a EUA) un número significativo de los negocios dirigidos a los consumidores estadounidenses (servicios médicos, farmacias, restaurantes, entre otros).

Las restricciones fronterizas, la inseguridad y el miedo a cruzar a México están conduciendo a la ruptura, pérdida o debilitamiento de su ca- 
rácter transfronterizo (reducción de los cruces de una lado a otro, blindaje de los espacios de celebración, cancelación de eventos). En este sentido, los desfiles internacionales pierden su "internacionalidad" en el mismo momento que los participantes de euA dejan de cruzar al lado mexicano, como ocurrió en Matamoros-Brownsville y Ciudad AcuñaEagle Pass en 2010 y 2011 respectivamente.

Asimismo, algunas personalidades invitadas se niegan a participar en los eventos mexicanos, como es el caso del Mister Amigo, título otorgado por Brownsville (TX), al que en 2009, 2010 y 2011 no asistió a Matamoros (TS).

Incluso algunas fiestas desaparecen parcial o completamente (Camargo y Miguel Alemán). Los lugares de celebración también se transforman con el incremento de la presencia de los cuerpos de seguridad y el cierre de los accesos a los puentes internacionales, sí como por el traslado a otros espacios percibidos como más seguros.

Debido al temor de los visitantes de Texas por la inseguridad, en 2009 en Reynosa (TS), los actos que siguen a la ceremonia del abrazo se cambiaron de ubicación, de la Plaza principal, en la zona centro, a la Plaza de la república, justo enfrente del paso fronterizo con Hidalgo (TX). ${ }^{6}$

Estrategias similares se aplican también en EUA: en Eagle pass (TX) desde 2011 se refuerza la vigilancia policial del lugar de celebración del carnaval internacional, Shelby park, en las inmediaciones de la línea fronteriza. A todo esto debe añadirse la reducción del número de cruces a México por compras, ocio y sanidad, que afecta a la economía regional de la zona fronteriza, agravada también por la crisis global de 2009.

En reacción a ello, algunas fiestas, en especial las organizadas en las ciudades mexicanas, se refuerzan a nivel simbólico con el doble objetivo de contrarrestar la percepción de inseguridad e incentivar el turismo. Incluso entre 2011 y 2012 se crean tres nuevas fiestas, con ceremonia del abrazo incluida: Ojinaga-Presidio, Río Bravo-Donna y, caso excepcional, una segunda entre Nuevo Laredo y Laredo; a estas puede añadirse el Friendship festival de Granjeno, desde 2007.

\section{Representaciones espaciales en la ceremonia del abrazo en el actual contexto fronterizo}

Después de haber identificado la evolución de las fiestas, se exponen los principales componentes de las representaciones sobre los espacios transfronterizos o fronterizos del río Bravo/Grande que se (re)producen en la

\footnotetext{
${ }^{6}$ En 2013 el Ayuntamiento de Reynosa programó de nuevo los actos en la Plaza principal, con el objeto de demostrar la mejora del estado de seguridad.
} 
ceremonia del abrazo en el contexto del cuarto periodo, es decir, del endurecimiento fronterizo. La relevancia de este evento reside en el hecho que se verbalizan y escenifican los dos tipos de representaciones -de unidad transfronteriza y de separación fronteriza- en los discursos y actitudes de las autoridades (locales, estatales, federales y otras) e invitados especiales, en el protocolo y en el lugar de celebración (los puentes y la guardarraya).

Por lo general cada una de las representaciones se asocia a un espacio geográfico diferente. Por un lado, la unidad transfronteriza con los pares de ciudades y, en una escala superior, el conjunto de la franja fronteriza de ambos países (lo que se ha llamado en ocasiones el tercer país y la tercera nación). Por el contrario, la separación fronteriza se asocia a los ámbitos nacionales, es decir México y EuA.

\subsection{Unidad transfronteriza}

\subsubsection{Unidad geográfica: una región geográfica, el río y los puentes}

Ya sean los pares de ciudades por separado o la franja fronteriza en su conjunto se presentan como una región singular, no por oposición al resto de México y de euA sino por aglutinar a ambos. La proximidad geográfica a "el otro lado" y la lejanía y aislamiento respecto a las respectivas capitales estatales y federales lo habría propiciado. La especificidad es explicada por la interdependencia, la adaptación a la frontera y a "el otro lado", así como a la participación simultánea en las espacialidades asociadas a ambos países.

Como se pone de manifiesto en las ceremonias, en ocasiones este reconocimiento se explicita con una denominación genérica para el conjunto regional, como es la frontera o, en el caso específico TamaulipasTexas, el Valle o la Región (del río Bravo/Grande). En cambio, suele ser más frecuente, sobre todo en los parlamentos de las autoridades texanas, referirse al espacio conceptual del "tercer país". Como se ha expuesto al inicio, se trata de una concepto ideal referido a la región extendida a ambos lados de la frontera (de delimitación variable: desde una anchura de 15 kilómetros por lado a la totalidad de los estados fronterizos), caracterizada por la hibridación cultural, la simbiosis y una creciente convergencia, mayor a la que pueda haber con los países respectivos.

Esta noción, aparecida en los años ochenta, fue ampliamente difundida por los medios de comunicación, aunque tanto en México como en EUA generó cierta polémica (como el debate académico antes señalado); en cambio fue recibida con buena aceptación en el ámbito artístico y cultural, incluidas las fiestas transfronterizas. 
Resulta interesante hacer notar que justamente se construyó a partir de la experiencia de la frontera México-Texas, y no del conjunto de la frontera México-Estados Unidos (Anzaldúa, 2007; Arreola y Curtis, 1993). Acorde con lo apuntado, este tercer país se justifica por ser el lugar de encuentro y la suma de "lo mejor de las dos naciones". 7 La acotación refleja claramente la idealización, muchas veces ingenua y utópica, sobre la integración del espacio transfronterizo, obviando u ocultando los contrastes, las inequidades y la asimetría de poder entre ambos países. En este sentido, no es de extrañar que sea principalmente pensada desde el lado estadounidense.

Tanto los discursos como el emplazamiento de la ceremonia colocan al río Bravo/Grande como elemento geográfico de unión; en tanto que este curso fluvial es compartido, ha moldeado el paisaje regional y constituye un recurso y un espacio fundamental. A la vez, la presencia e identificación con el río permite a las comunidades fronterizas distinguirse del resto de la frontera México-Estados Unidos (el sector comprendido entre Ciudad Juárez-El Paso y Tijuana-San Diego) e, incluso, de una gran parte de los espacios transfronterizos del mundo, al ser una de las fronteras fluviales más largas. El río se construye, en este sentido, como símbolo de la identidad transfronteriza, expresada como binacional: "La fiesta [...] reafirma la identidad binacional de nuestro querido río Bravo". ${ }^{8}$

Los puentes constituyen también un elemento de unidad geográfica de primera magnitud; ya se ha expuesto que son el lugar físico y simbólico de celebración, pero además constituyen la demostración de la proximidad geográfica y de los esfuerzos tecnológicos y urbanísticos para conseguirla. En este sentido, en los discursos se suele expresar que son las infraestructuras que facilitan y representan las comunicaciones, los flujos, la amistad y la comprensión entre ambos lados, así como los símbolos de unión entre las ciudades hermanas.

\subsubsection{Unidad sociocultural: lazos de amistad y de parentesco, y cultura propia}

La unidad sociocultural se justifica en el reconocimiento y reafirmación de los lazos de amistad y familiares, sobre todo, entre los habitantes de ambos lados de la frontera. Ello se expresa verbalmente y por medio del abrazo, por lo que acarrea este gesto de intimidad y familiaridad.

\footnotetext{
${ }^{7}$ Declaración de Alan Bersin, representante especial para los Asuntos Fronterizos del Department of Homeland Security de Estados Unidos en la ceremonia del abrazo Laredo-Nuevo Laredo, 2010 (López, 2010).

${ }^{8}$ Declaración de Juan Diego Guajardo, alcalde de Río Bravo (TS), ceremonia del abrazo Río Bravo-Donna, 2012. (Reynosa News, 2012).
} 
En muchos casos los lazos se establecieron desde la fundación misma de las ciudades (Kearney y Knopp, 1995), por lo que la unidad se interpreta como algo tradicional e histórico y cuyo elemento central y fundamental lo constituyen las familias. En este sentido, la unidad está sujeta a que éstas mantengan vivas sus relaciones y convivencia a ambos lados de la frontera. Ello incluye, en cierto sentido tautológicamente, la asistencia y participación en las fiestas transfronterizas, en tanto que son un evento tradicional y familiar.

En esta dirección se entienden afirmaciones como "Yo recuerdo venir a Nuevo Progreso con mis padres, de compras y pasarla muy bien" expresadas durante los parlamentos. Aquel reconocimiento es a la vez una advertencia, puesto que si se perdieran las relaciones implicaría quizá una separación más dura que la que inflige la frontera material y jurídica.

En una dirección similar, la unidad también se apoya en haber identificado y reconocido una cultura transfronteriza propia y compartida en ambos lados, la cual se transmite y reproduce gracias a aquellos vínculos sociales y familiares.

Esta cultura se diferenciaría de lo estrictamente mexicano y norteamericano, pero a la vez sería resultado de la hibridación de elementos. Los llamados durante la ceremonia del abrazo se refieren principalmente a un estilo de vida y gustos (música, gastronomía, hábitos de consumo, ocio y religiosidad) y en menor medida a aspectos como la etnicidad (mayoritariamente mexicana en ambos lados) y la lengua (el español y -como resultado de la hibridación- el spanglish). Sin embargo, en las ceremonias no se da tanta importancia al hecho de la hibridación como a su excepcionalidad, en tanto que difiere de las lógicas de carácter nacional asumidas.

Esto nos lleva a notar el origen paradójico de la conceptualización y definición de esta cultura transfronteriza. Para ello se parte de la noción de correspondencia entre unidades geopolíticas y culturales; es decir, que a México le correspondería una única cultura mexicana, y a EuA una única cultura estadounidense, culturas que sólo existirían dentro de los límites políticos de cada país y en cada país no podría caber más que una. Esta noción constituye uno de los errores metageográficos identificados por Lewis y Wigen (1997).

Por otra parte, la identificación de una cultura en las regiones fronterizas de ambos países contradice aquella correspondencia y exclusividad. La solución a esta paradoja es, de nuevo, el concepto de tercer país o tercera nación, el cual permite asumir, sin entrar en contradicción, la

\footnotetext{
${ }^{9}$ Declaración de Sara Todler, representante de Rio Grande Valley Partnership Chamber of Commerce, ceremonia del abrazo Nuevo Progreso-Progreso, 2012. (Reynosa News, 2102a).
} 
correspondencia entre países y culturas. No obstante, como Dear (2013) advierte después de haber observado otras prácticas espaciales en la frontera México- EUA, el simple reconocimiento no basta, puesto que no ha conducido por ahora a expresar explícitamente la existencia de dicha tercera nación ni, menos aún, a identificarse únicamente con ella. En este sentido, se produce un fenómeno similar al de la unidad geográfica, donde se da la ausencia de una clara e integradora denominación regional.

\subsubsection{Unidad económica: la economía regional, el comercio interamericano $y$ los puentes}

Las relaciones e interdependencias económicas de las ciudades hermanas es otro de los principales componentes discursivos. Probablemente su peso esté sobredimensionado debido a los objetivos económicos de las fiestas, en tanto que se convierten en aparadores para la promoción de las ciudades (turismo, comercio y márquetin territorial para la captación de inversiones en industria y logística).

En relación al turismo de compras y de salud, por ejemplo, la referencia a los winter texans resulta casi obligatoria, tanto para subrayar su impacto económico a ambos lados de la frontera como para incentivar que continúen visitándola cada ańo. Como se desprende de este mismo ejemplo, la representación de la unidad no sólo toma en cuenta las interrelaciones entre los espacios regionales fronterizos, sino que también lo hace a una escala mayor. En ocasiones el espacio de referencia es la totalidad de los dos países o el conjunto de América del Norte. En segundo lugar, la unidad económica se justifica y refleja también en los puentes internacionales, por donde cruzan los flujos comerciales y laborales, tanto de nivel global como local, legales e ilegales (aunque éste último no se explicite en los parlamentos).

\subsubsection{Unidad sociopolitica: superando la asimetría de poder (la cooperación transfronteriza, frente común a la inseguridad y rechazo al muro fronterizo)}

Al tener en cuenta la asimetría de poder en las relaciones México- EUA, en la ceremonia del abrazo se representa la voluntad de superarla, mostrando una igualdad o equiparación que se transmite desde los parlamentos de las autoridades, el protocolo y la escenificación del espacio de celebración. La primera muestra de ello es el propio abrazo entre autoridades políticas, militares, económicas y religiosas. Es importante recordar que las autoridades de un lado sólo se abrazan con las equivalentes del otro. Asimismo, las banderas de ambos países se sitúan una al lado de la otra y al mismo nivel. En una dirección similar, el abrazo infantil 
tiene por objetivo transmitir a las nuevas generaciones un sentimiento de igualdad y fraternidad, alejado de los prejuicios y rencores que podían acarrear las anteriores.

De todo ello se deriva la necesidad de expresar una acción política conjunta. Tres ejemplos son representativos de ello. En primer lugar, la constatación, la voluntad o la promesa de cooperar entre las ciudades hermanas para el mutuo beneficio. De hecho, a menudo se presentan las fiestas como prueba de estas relaciones de cooperación o como el espaciotiempo que permite plantearlas y consensuarlas. Más allá de estas características generales, cada par de ciudades expone sus casos particulares; para citar sólo algunos: la coordinación de las acciones en materia de desarrollo comunitario entre Reynosa (TS) e Hidalgo (TX), la acción conjunta en el ámbito de las infraestructuras de transporte entre Nuevo Laredo (TS) y Laredo (TX), y la programación de reuniones periódicas entre las alcaldías de Río Bravo (TS) y Donna (TX).

En segundo lugar, en el actual contexto fronterizo, la unidad y la cooperación transfronterizas están condicionadas por la inseguridad y la violencia asociada a los flujos comerciales ilícitos, que a la par afectan $-y$ así se reconoce- a la vida cotidiana y a la economía de ambos lados de la frontera. Frente a esto, con la ceremonia y en general con las fiestas se desea exhibir un fortalecimiento de dicha unidad y cooperación.

Una actitud similar se muestra, en tercer y último lugar, frente a la violencia institucional que suponen las políticas fronteriza y migratoria aplicadas por EUA. En esta dirección se verbalizan mensajes como: "estamos demostrando que no existen las fronteras, no hay límites entre nosotros". ${ }^{10}$ A pesar de la retórica utilizada, no se trata tanto de negar la existencia de la frontera como de oponerse a su endurecimiento material y jurídico. Desde 2007, año en el que se inició la construcción del muro fronterizo en Texas, esto se ha traducido en un rechazo argumentado en la interrelación entre ambos lados, los elementos de unión (como los puentes) y en la ineficacia para reducir la migración indocumentada y el narcotráfico. En relación a este último argumento se reclama a las autoridades federales estadounidenses que "se deben buscar otras alternativas"11 que sean efectivas y no afecten a la cotidianidad de y entre las dos riberas.

\footnotetext{
${ }^{10}$ Declaración de Everardo Villarreal, alcalde de Reynosa (TS), ceremonia del abrazo ReynosaHidalgo, 2012 (Peña, 2012).

${ }^{11}$ Declaración de John David Franz, mayor de Hidalgo (TX), ceremonia del abrazo ReynosaHidalgo, 2008 (Wradio, 2008).
} 


\subsubsection{Unidad histórica: una ausencia destacable}

A pesar de que ambos lados habían formado parte históricamente de un único espacio étnico (la Gran Chichimeca o la Comanchería, como lo llamaron los colonizadores españoles, o la Aridoamérica, concepto antropológico reciente) y político (la Nueva España y, después, México), ni en los parlamentos ni en la escenificación de la ceremonia se hace referencia alguna. La casi única excepción se da en la ceremonia entre Laredo (TX) y Nuevo Laredo (TS), donde ondean las "Siete banderas de Laredo", que incluye las de España, México y la República del Río Grande, ${ }^{12}$ aunque culmina con la de Estados Unidos.

Se trata además de una representación institucionalizada propia de la ciudad de Laredo (a semejanza de las "Seis banderas de Texas"), reflejada, por ejemplo, en su escudo; no es representativa, por lo tanto, del espacio transfronterizo. Se detecta en este sentido la ausencia de una representación de unidad histórica que contrasta con su presencia en las fiestas de la frontera México-California, como en la Fiesta del Río de Imperial Beach y Tijuana (Conway, 2010). La diferencia entre ambos sectores fronterizos, en cuanto a la ausencia o presencia de la representación de unidad histórica, se pueda deber a la diferente historia de estos sectores. La formación de la frontera México-Texas fue mucho más violenta que no la del sector occidental, dramática para México y patriótica para Estados Unidos. La ausencia y la historia violenta nos conducen directamente a las representaciones de separación fronteriza.

\subsection{Separación fronteriza}

\subsubsection{Separación geográfica y politica: la frontera, las restricciones de paso y la violencia}

La línea fronteriza es omnipresente en las ceremonias del abrazo. En contraste con el simbolismo de los puentes, la ubicación también coincide con la guardarraya entre ambos países. Inevitablemente, el escenario y el telón de fondo de los abrazos son los elementos físicos de la frontera: la barda - por lo general, construida a partir de 2007-, las rejas, el puerto de entrada, la aduana, la patrulla fronteriza y los agentes de migración. Aunque su presencia es difícil de ocultar para las fotos oficiales y, en cierta medida, las publicadas en los medios de comunicación se utilizan

\footnotetext{
${ }^{12}$ La República del Río Grande fue un intento, en 1840, de formar una nación-estado independiente compuesta por Coahuila, Nuevo León y Tamaulipas, que en el aquel momento se extendían hasta el río Nueces (en disputa a su vez con la República de Texas), y cuya capital debía ser Laredo (Guerrero, 2002).
} 
encuadres donde aquellos elementos estén ausentes o resulten menos visibles. Si en ocasiones se quiere tapar, en otras la frontera se celebra como un logro positivo de la humanidad y de la civilización: "Es un honor [...] estar en esta línea que significa tanto y que un día pintaron los humanos para organizarnos y funcionar como sociedad". ${ }^{13}$ En uno y otro caso, se busca olvidar que la frontera fue resultado de dos conflictos bélicos, la guerra de Independencia de Texas (1835-1836) y la guerra México-Estados Unidos (1846-1848).

Resultado y reflejo de la asimetría de poder, esta celebración y olvido no son iguales para ambos lados de la frontera: para EUA es una forma de no cuestionar el pasado y perpetuar su dominio en el presente. En esta misma dirección, Van Wagenen (2012) muestra como las consecuencias de dichos conflictos se han olvidado convenientemente en EUA, mientras que en México continúan estando muy presentes en el imaginario nacional, donde, por ejemplo, la pérdida de una parte de su territorio es un tema casi cotidiano.

La frontera y, sobre todo, el poder ejercido por EUA también se manifiestan en las restricciones de paso impuestas a la población mexicana para cruzar al otro lado, lo que dificulta o incluso impide, como ya se ha apuntado, su asistencia a los eventos del lado estadounidense. De ello no están exentos ni los invitados especiales, como cuando en 2006 la U.S. Customs and Border Protection (Oficina de Aduanas y Protección Fronteriza) no permitió acceder a Laredo (TX) a los invitados procedentes de Nuevo Laredo (TS).

Las restricciones - permanentes, antes, durante y después de las fiestas- convierten al río y a los puentes en un símbolo de división: en el río mueren ahogadas personas cuando intentan cruzar sin documentos, y en los puentes se forman diariamente largas filas para poder entrar a EUA debido a la revisión de la documentación, del equipaje o de los automóviles.

En sentido inverso, las alertas de seguridad emitidas por los consulados de euA en México contribuyen también a disminuir el número de cruces y a aumentar la percepción de inseguridad y el miedo al lado mexicano. En algunas ocasiones las alertas han afectado directamente al desarrollo de las fiestas, como en el caso de Matamoros-Brownsville en 2013. Dos días antes de iniciarse las festividades, el Consulado de Estados Unidos en Matamoros (TS) emitió un aviso recomendando a los ciudadanos estadounidenses no viajar a Tamaulipas si no era estrictamente necesario. Paradójicamente, el Cónsul general estuvo presente en varios de los actos

${ }^{13}$ Declaración de Luis Padua, invitado especial, ceremonia del abrazo Río Bravo-Donna, 2013 (Sánchez, 2013). 
de las Fiestas mexicanas de Matamoros, además de la ceremonia del abrazo, donde su presencia es requerida por el carácter diplomático del evento.

Por otra parte, la voluntad mostrada contra la violencia queda en entredicho frente a la inacción de las autoridades en la resolución de estas problemáticas, ya sea por falta de voluntad real o de capacidad políticoadministrativa. Muestra de ello lo ofrece la respuesta a la protesta civil que, en 2006, se llevó a cabo en Laredo (TX) poco antes del inicio de la ceremonia, en el acceso al puente internacional (Peńa, 2006). Los convocantes reivindicaban las personas desaparecidas y secuestradas en Nuevo Laredo (TS), los cuales fueron completamente ignorados por las autoridades asistentes a la ceremonia. De la situación de inseguridad en México también es indicativo que dicha protesta se celebrara en el lado estadounidense.

\subsubsection{Separación sociocultural y politica: identidad y simbolos nacionales}

Las fiestas también son un acto de reafirmación nacional respeto a "el otro", para marcar y reforzar las diferencias e identidades de euA y México. Todas las ceremonias del abrazo están presididas por las respectivas banderas nacionales y, con pocas excepciones, por la entonación de los respectivos himnos. Es habitual el intercambio de banderas entre las diferentes autoridades y entre las parejas de niños y niñas; estos últimos, además, son vestidos con los trajes considerados, desde un punto de vista esencialista, tradicionales de cada uno de los países (aunque también de la región). Asimismo, como se ha apuntado, se invitan y asisten cargos políticos y militares estatales y federales. Banderas, himnos y cargos trasladan a las fiestas el nivel nacional, desplazando a segundo término el local.

Otras formas de identificación nacional se transmiten mediante los motivos y significados particulares de celebración. Como ya se ha expuesto, la ШвC de Laredo fue en sus orígenes un acto de enaltecimiento de EUA; el de la Border fest de Hidalgo fue para conmemorar el bicentenario.

Desde la década de 1960 se puede decir que la wBC es una fiesta con componentes de ambos países que continúa incluyendo actos netamente de reafirmación de la identidad nacional y angloamericana, tal es el caso del baile de la Society of Martha Washington y el desfile de la Princesa Pocahontas. En la ceremonia del abrazo se encarnan a George Washington y Miguel Hidalgo, dos de los mitos fundacionales de EuA y México, que se abrazan e intercambian banderas.

De forma similar, el Día del turista de Nuevo Progreso coincide con el natalicio de Benito Juárez (el 21 de marzo), otro de los referentes nacionales mexicanos; aunque actualmente no se le hace homenaje especial alguno, sí se brindaron homenajes en las ceremonias celebradas en 2010, 
en coincidencia con el bicentenario de la independencia de México y el centenario de la Revolución Mexicana.

Por último, si al hablar de la unidad transfronteriza se ha apuntado que la lengua (el español a ambos lados de la frontera) no tiene una presencia destacada para argumentar la cultura compartida, en este caso se usa (el español y el inglés) para marcar y subrayar las diferencias.

Aunque la etnicidad mayoritaria en los condados fronterizos de EUA sea la mexicana (más de 50\% de la población según el 2010 United States Census) y que el español sea ampliamente usado, el inglés es la lengua de las autoridades estadounidenses en la ceremonia. Incluso se da el caso que empiezan los parlamentos en español y poco después cambian al inglés, como le ocurrió al alcalde de Brownsville (TX) durante la ceremonia con Matamoros (TS) de 2013.

El uso del inglés también se debe a dos situaciones que, una vez más, marcan las diferencias (Richardson, 1999; Villa, 2000): primero las comitivas de las ciudades texanas están integradas por autoridades de etnicidad angloamericana, en ocasiones en una proporción mayor a la esperada por el perfil demográfico (especialmente en las fiestas más antiguas, Laredo y Brownsville); segundo, las autoridades mexicoamericanas se anglicanizaron como mecanismo de ascensión social y de diferenciación del mexicano estigmatizado étnica y socialmente.

\section{Conclusiones}

Conviene recordar que, con el propósito de determinar qué representaciones espaciales se (re)producen en el marco espacio-temporal de lo que se ha definido como fiestas transfronterizas, se han analizado las fiestas de las ciudades hermanas de la frontera del río Bravo/Grande (México- EuA). Se ha procedido a identificar los componentes discursivos de los parlamentos de las autoridades y de los lugares de la ceremonia del abrazo, uno de los actos más significativos de las fiestas, en lo que se refiere a la participación de autoridades de ambos países y por celebrarse en la guardarraya.

Dichos eventos son, en tanto que prácticas espaciales, una apropiación y transformación temporal de los espacios fronterizos -en este caso, los puentes internacionales que conectan las ciudades hermanas- por parte de las autoridades participantes (representantes de los gobiernos locales, estatales y federales, y representantes económicos, sociales, militares y religiosos de ambos lados de la frontera).

No se debe olvidar que los puentes, además de su dimensión material, poseen un peso simbólico destacado, desde el nivel local al universal. Por medio de aquella apropiación y de los parlamentos se transmite un conjunto de representaciones relativas a la región transfronteriza del río 
Bravo/Grande y a los países que la frontera divide (México y EUA). La adscripción de las autoridades acarrea un sesgo sociopolítico particular y determina las características de las representaciones, que podrían diferir de las de otros grupos sociales (que no participan o están excluidos).

De acuerdo al marco teórico, del análisis se desprende que en la ceremonia del abrazo se proyectan dos tipos de representaciones espaciales, a priori contradictorias entre sí. Una, de unidad transfronteriza, basada en la idea de compartir la región del río Bravo/Grande (el río, los puentes, los amigos y la familia, una cultura híbrida, la economía y un proyecto político) y que se puede sintetizar en el concepto de tercer país o tercera nación. Su construcción se apoya en un imaginario de integración transfronteriza, hibridación, igualdad, amistad y de reducción de las fricciones causadas por la frontera. No obstante, esta interpretación puede ser calificada de ingenua o utópica, en tanto que esconde las desigualdades y la asimetría de poder entre euA y México.

En este sentido, la unidad transfronteriza constituye una representación asumible y conveniente para el grupo social y el país que domina las relaciones desiguales y asimétricas, en este caso EUA. En consecuencia, la unidad transfronteriza, contrario a lo que representa, viene a perpetuar aquellas relaciones. Esto nos conduce directamente al segundo tipo de representaciones, de separación fronteriza, por el cual se reafirman los espacios e identidades nacionales. Esta segunda, obviamente, tampoco se construye en un plano de igualdad, sino que lo hace en el contexto de asimetría de poder, la cual se ve amplificada por la posición sociopolítica de los grupos sociales participantes, en su mayoría autoridades políticas. Para matizar aún más estas dos representaciones es necesario situarlas en el marco de dialéctica entre lo local y lo nacional y entre la integración y la fortificación.

No debe sorprender que los elementos de unidad transfronteriza sean trasmitidos mayoritariamente por las autoridades locales en sus parlamentos, mientras que los de separación se expresan mediante el protocolo, el escenario de celebración y en los parlamentos de las autoridades de nivel estatal y federal.

De todo ello se deduce que la oposición entre ambas representaciones es más aparente que real y que son, en cierta forma, complementarias. Inicialmente, porque ambas son (re)producidas simultáneamente y de forma imbricada; luego porque la unidad transfronteriza no es vista como una amenaza por las autoridades que representan a los estados-nación; por el contrario, impide cuestionar o permite perpetuar la asimetría de poder. Finalmente, las autoridades locales no ponen en cuestión a los Estados-nación; a lo sumo, las decisiones políticas nacionales que les 
afectan, como en el caso de la construcción del muro fronterizo por parte de EUA.

La dualidad está presente en todos los componentes que constituyen las representaciones espaciales. Se pueden destacar dos elementos geográficos: el río y los puentes internacionales. El río constituye el nexo entre ambos lados, mientras que los puentes permiten y facilitan todo tipo de relaciones (sociales y económicas), además de ser el símbolo por excelencia de la buena vecindad, la amistad, el acercamiento y la comprensión. A la vez, el río y los puentes son material y simbólicamente la frontera que separa ambos lados. El curso fluvial es un obstáculo físico a salvar y marca la línea divisoria, endurecida por la aplicación por parte de eUA de políticas fronterizas y migratorias, que se concentran y se intensifican en los puertos de entrada, coincidentes con los puentes.

Esta dicotomía se detecta también entre los niveles/escala local y nacional. Es local la organización y celebración de las fiestas y las representaciones de unidad transfronteriza, pero por el contrario el protocolo traslada a estos espacios el nivel nacional. El propio abrazo constituye la primera muestra de ello, ya que emula el gesto que protagonizaron los presidentes de EuA y México en 1960 en Ciudad Acuña (CO), aunque a la vez simbolizan un mayor acercamiento y familiaridad, sobre todo en comparación con el apretón de manos al que sustituyó.

En la misma dirección se emplazan los motivos coyunturales de algunas de las fiestas, en tanto que son una reacción local a políticas nacionales (el rechazo a la construcción del muro fronterizo, la defensa de los derechos de la población mexicoamericana en EUA y la promoción de las relaciones amistosas y de buena vecindad). En otras ocasiones no sólo se trata de una equiparación, sino de una suplantación del espacio local por parte de los niveles nacionales. En esta dirección sobresale el protagonismo otorgado a los símbolos e identidades nacionales, legitimadas por la presencia de autoridades políticas y militares de nivel estatal y federal. En cierto sentido se puede decir que su (re)producción crea unos efectos tanto o más insidiosos que el propio endurecimiento material y legal de la frontera.

Cuando ambas representaciones entran en clara contradicción, esta suplantación llega a anular la unidad transfronteriza, sobre todo cuando aquélla implicaría cuestionar las relaciones asimétricas entre ambos Estadosnación. Resulta significativa en este sentido la ausencia de un discurso de unidad histórica. Mientras que el río, los puentes, los flujos de cualquier tipo y motivo o la cultura no cuestionan la actual delimitación fronteriza entre México y EUA, cualquier referencia a un espacio geopolítico común pasado, especialmente a cuando ambos lados del río formaban parte 
constituyente de México, sí lo hace; de hacerlo, implícitamente se pondría en cuestión la anexión de Texas y la guerra que definió dicha delimitación.

De forma similar también se ha procedido a una reinvención de la historia, como ocurre en los festejos de Laredo en honor a George y Martha Washington y Pocahontas, personajes históricos anteriores a la incorporación de Texas a EUA, provocando así una discontinuidad histórica entre ambos lados.

La investigación perseguía un segundo objetivo, derivado de la constatación que tanto las fiestas como las representaciones espaciales responden a los contextos históricos de la frontera donde se ubican. En este sentido, se ha prestado una atención particular al actual contexto de endurecimiento fronterizo, que se inicia, orientativamente, en 2006 y caracterizado por una política fronteriza más restrictiva por parte de EUA y una situación de violencia e inseguridad en México, lo que a priori está produciendo un debilitamiento e, incluso, ruptura de las relaciones transfronterizas.

Se ha mostrado que el desarrollo de las fiestas ha sido afectado tanto por la violencia asociada al narcotráfico, al crimen organizado y a la guerra en México contra estos grupos como por el endurecimiento legal y material de la frontera por parte de EuA. De nuevo la respuesta es doble: la inseguridad y el endurecimiento están provocando una pérdida del carácter transfronterizo de las fiestas (principalmente por la ausencia en el lado mexicano de grupos e individuos procedentes del estadounidense $\mathrm{y}$ de restricciones de internamiento a EUA).

A pesar que en este periodo han desaparecido algunas fiestas y se han incrementado las medidas de seguridad y restricciones en los lugares de celebración (en algún caso, con cambios de localización), la mayoría se han mantenido e incluso se han creado de nuevas. Asimismo, en el marco de las fiestas los pares de ciudades han constituido simbólicamente un frente común, donde destaca el reconocimiento, por parte de las autoridades locales de ambos lados, de las consecuencias que esta situación tiene para los espacios fronterizos. Sin embargo, más allá de esta muestra de buenas intenciones no existe una voluntad o una capacidad política real de resolución de las múltiples problemáticas.

\section{Fuentes consultadas}

Anzaldúa, Gloria (2007), Borderlands/La Frontera, Aunt Lute Books, Jackson. 
Arreola, Daniel D. (2013), Postcards from the Rio Bravo border. Picturing the place, placing the picture, 1900s-1950s, University of Texas Press, Austin.

Arreola, Daniel D. y James R. Curtis (1993), The mexican border cities. Landscape anatomy and place personality, The University of Arizona Press, Tucson.

Benedetti, Alejandro (2007), "La región circumpuneña. Algunas consideraciones para iniciar el debate", en A. Corder (ed.), Actas del VI Seminario internacional de integración sub-regional: sociedades de frontera, montaña y desierto, Ediciones del Instituto de Estudios Internacionales, Iquique, pp. 14-26.

Bhabha, Homi (1990), "The third space”, en J. Rutherford (ed.), Identity, community, culture, difference, Lawrence and Wishart, London, pp. 207-221.

Breugnot, Jacqueline (2012), "La construction des espaces frontaliers européens: entre dynamisme et résistance", Alterstice. Revue Internationalle de la Recherche Interculterelle, 2 (1), Université Laval, Québec, pp. 67-78.

Brunner, José J. (1992), América Latina: cultura y modernidad, Grijalbo, México.

Bustamante, Jorge A. (2012), Sembrar en el desierto. Crónicas de los primeros 30 años de El Colegio de la Frontera Norte, El Colegio de la Frontera Norte, Tijuana.

Carmin, Joann, Barbara Hicks y Andreas Beckmann (2003), "Leveraging local action. Grassroots initiatives and transboundary collaboration in the formation of the White Carpathian Euroregion", International Sociology, 18 (4), Sage, London, pp. 703-725.

Conway, Frederick J. (2010), "Imaginando la Cuenca del Río Tijuana", Culturales, 6 (12), Universidad Autónoma de Baja California, Mexicali, pp. 75-96.

Dalstrom, Matthew D. (2012), "Winter Texans and the re-creation of the american medical experience in Mexico", Medical anthropology: cross-cultural studies in health and illness, 31 (2), Routledge, London, pp. 162-177. 
Dávila, Rosaura (2013), De carnaval a fiestas mexicanas, Coatlicue, Matamoros.

Dear, Michael (2013), Why walls won't work: repairing the US-Mexico Divide, Oxford University Press, New York.

De Bres, Karen y James Davis (2001), "Celebrating group and place identity: a case study of a new regional festival”, Tourism Geographies, 3 (3), Routledge, London, pp. 326-337.

Dennis, Dion (1997), "Washington's birthday on the Texas border", Ctheory, 169, <http://www.ctheory.net/articles.aspx?id=169>, 6 de marzo de 2013.

De Rapper, Gilles (2010), "Religion on the border: sanctuaries and festivals in post-communist Albania", en Galia Valtchinova (ed.), Religion and boundaries. Studies from the Balkans, Eastern Europe and Turkey, Isis Press, Istanbul, pp. 247-265.

Diamond, Jared (2012), The world until yesterday: what can we learn from traditional societies?, Viking Press, New York.

Dorsey, Margaret E. y Miguel Díaz-Barriga (2010), "Beyond surveillance and moonscapes: an aternative imaginary of the Us-Mexico border wall", Visual Anthropology Review, 26 (2), American Anthropological Association, Arlington, pp. 128-135.

Fernández, Anna y Lilia Venegas (2010), "Fiesta, identidad y estrategias de una minoría que se organiza: la reina de las flores de una comunidad latina en Texas", Migraciones internacionales, 5 (3), El Colegio de la Frontera Norte, Tijuana, pp. 113-142.

Fernández de Casadevante, Carlos (1989), La frontière franco-espagnole et les relations de voisinage, Harriet, Bayonne.

Eagles, Munroe (2010), "Organizing across the Canada-us border: binational institutions in the Niagara region", American Review of Canadian Studies, 40 (3), Routledge, London, pp. 379-394.

García Canclini, Nestor (2003), "Malentendidos interculturales en la frontera México-Estados Unidos”, en José Luis García y Ascensión 
Barañano (coords), Culturas en contacto. Encuentros y desencuentros, Ministerio de Educación, Cultura y Deporte, Madrid.

Getz, Donald (2010), "The nature and scope of festival studies", International Journal of Event Management Research, 5 (1), The University of Queensland, Brisbane, pp. 1-47.

Guerrero, Antonio (2002), La república del Río Grande y la crónica del pais que nunca existió: 1837-1840, Universidad de Monterrey, Monterrey.

Green, Stanley (1992), A history of the Washington Birthday Celebration, Border Studies Publishing, Laredo.

Grimson, Alejandro (2000), "El puente que separó dos orillas. Notas para una crítica del esencialismo de la hermandad”, en Grimson, Alejandro (comp.), Fronteras, naciones e identidades: la periferia como centro, Ciccus-La Crujía, Buenos Aires, pp. 201-231.

Grimson, Alejandro (2004), "Las culturas son más híbridas que las identificaciones. Diálogos inter-antropológicos”, ponencia presentada en Reflections on the future, University of California, 20-21 de febrero, Santa Cruz.

Hamman, Philippe (2003), "La coopération intercommunale transfrontalière: vers une nouvelle gouvernance locale en Europe?", Pyramides, 7, Centre d'Etudes et de Recherches en Administration Publique, Bruselas, pp. 183-202.

Hinfray, Noémie (2007), "Les processus de construction des territoires transfrontaliers en Europe: éléments d'analyse”, Mosella, 32 (1-4), Université de Lorraine, Metz, pp. 79-95.

Janiskee, Robert (1996), "Community festivals in the Carolinas", en G. Gordon Bennett (ed.), Snapshots of the Carolinas: landscapes and cultures, Association of American Geographers, Washington, pp. 57-61.

Kearney, Milo y Anthony Knopp (1995), Border cuates. A history of the us-Mexican twin cities, Eakin Press, Austin. 
Klein, Alan M. (1997) Baseball on the border: a tale of two Laredos, Princeton University Press, Princeton.

Knopp, Anthony, Manuel F. Medrano y Priscilla Rodriguez (2009), Charro Days in Brownsville, Arcadia Publishing, Charleston.

Lewis, Martin W. y Kären Wigen (1997), The myth of continents. A critique of metageography, University of California Press, Berkeley.

Lindón, Alicia y Daniel Hiernaux (dirs.) (2006), Tratado de Geografía Humana, Anthropos-Universidad Autónoma Metropolitana, Barcelona-México.

López, Primitivo (2010) "Eu y México co-responsables de problemática en frontera”, Hoy Tamaulipas, HoyTamaulipas.net, <http://www. hoytamaulipas.net/notas/3875/EU-y-Mexico-co-responsablesde-problematica-en-frontera-.html>, 2 de marzo de 2013.

Mathisen, Stein R. (2009), "Festivalising heritage in the borderlands: constituting ethnic histories and heritages under the Rule of the Finn Forest Republic", Journal of Ethnology and Folkloristics, 3 (2), University of Tartu, Tartu, pp. 13-31.

Migdal, Joel S. (ed.) (2004), Boundaries and belongings. States and societies in the struggle to shape identities and local practices, Cambridge University Press, Cambridge.

O'Dowd, Liam (2002), "The changing significance of european borders", Regional \& Federal Studies, 12 (4), Routledge, London, pp. 13-36.

Oliveras, Xavier (2013), "La cooperación transfronteriza en la Cerdanya (frontera España-Francia)", Boletín de la Asociación de Geógrafos Españoles, 62, Asociación de Geógrafos Españoles, Madrid, pp. $25-48$.

Paasi, Anssi (1996), Territories, boundaries and consciousness: the changing geographies of the finnish-russian border, Wiley, Chichester.

Pastor, Robert A. y Jorge G. Castañeda (1988), Limits to friendship: the United States and Mexico, Alfred A. Knopf, New York. 
Peña, Carlos (2012), "Alcalde texano da su último abrazo fraternal con México", En Linea Directa, <http://enlineadirecta.info/?option= view\&article=175932\#sthash.IRMQ6Quc.dpbs>, 29 de febrero de 2013.

Peña, Elaine (2006), “De-politicizing border space”, E-misferica. Performance and Politics in the Americas, 3 (2), <http://hemisphericinstitute.org/journal/3.2/eng/en32_pg_pena.html>, 6 de marzo de 2013.

Perkmann, Markus (2003), "Cross-border regions in Europe. Significance and drivers of regional cross-border co-operation", European Urban and Regional Studies, 10 (2), Sage, London, pp. 153-171.

Philo, Chris (1999), "Más palabras, más mundos: reflexiones en torno al 'giro cultural' y a la geografía social”, Documents d'Anàlisi Geogràfica, 34, Universitat Autònoma de Barcelona, Barcelona, pp. 81-99.

Prokkola, Eeva-Kaisa (2010), "Borders in tourism: the transformation of the Swedish-Finnish border landscape", Current Issues in Tourism, 13 (3), Routledge, London, pp. 223-238.

Reynosa News (2012), “Inician tradición con Festival del Río”, ReynosaNews.com, <http://reynosanews.com/info/2012/02/28/iniciantradicion-con-festival-del-rio/>, 29 de febrero de 2013.

Reynosa News (2012a), "Llegan miles de turistas a Nuevo Progreso", ReynosaNews.com, <http://reynosanews.com/info/2012/03/21/ llegan-miles-de-turistas-a-nuevo-progreso/>, 29 de febrero de 2013.

Quinn, Bernadette (2003), "Symbols, practices and myth-making: cultural perspectives on the Wexford Festival Opera”, Tourism Geographies, 5 (3), Routledge, London, pp. 329-349.

Quinn, Bernadette (2005), "Changing festival places: insights from Galway”, Social \& Cultural Geography, 6 (2), Routledge, London, pp. 237-252.

Richardson, Chad (1999), Batos, bolillos, pochos and pelados: class and culture on the south Texas border, University of Texas Press, Austin. 
Sánchez, Luis (2013), "Realizan alcaldes de Río Bravo y Donna fiesta tradicional", Hora Cero, http://www.horacero.com.mx/tamaulipas/realizan-alcaldes-de-rio-bravo-y-donna-fiesta-tradicional, 2 de marzo de 2013.

Soja, Edward (1996), Thirdspace: journeys to Los Angeles and other real and imagined places, Wiley-Blackwell, Hoboken.

Trillo, Juan M. y Ruben Lois (2011), "La frontera como motivo de atracción: una breve mirada a las relaciones Galicia-Região Norte", Geopolitica(s). Revista de estudios sobre espacio y poder, 2 (1), Universidad Complutense de Madrid, Madrid, pp. 109-134.

Unigarro, Daniel E. (2012), "Alegría sin fronteras entre Brasil, Colombia y Perú: de cómo 'tres países hermanos' celebran el nacionalismo", Maguaré, 26 (1), Universidad Nacional de Colombia, Bogotá, pp. 263-297.

Van Wagenen, Michael (2012), Remembering the forgotten war. The enduring legacies of the U.S.-Mexican War, University of Massachusetts Press, Amherst.

Vila, Pablo (2000), Crossing borders, reinforcing borders. Social categories, metaphors and narrative on the US-Mexico frontier, University of Texas Press, Austin.

Warnaby, Gary y Dominic Medway (2008), "Bridges, place representation and place creation”, Area, 40 (4), Royal Geographical Society, London, pp. 510-519.

Wilkinson, Jane (2007), Performing the local and the global: The Theatre estivals of Lake Constance, Peter Lang, Berna.

Wooldridge, Ruby y Robert B. Vezzetti (1989), “The founding of Charro Days", en Milo Kearney (ed.), More Studies in Brownsville History, University of Texas at Brownsville, Brownsville, p. 390-391.

Wradio (2008), "Reitera alcalde de Hidalgo, Texas, rechazo al muro fronterizo", WRadio, <http://www.wradio.com.mx/noticias/reitera-alcalde-de-hidalgo-texas-rechazo-al-muro-fronterizo/ 20080228/nota/556246.aspx>, 29 de febrero del 2013. 
Young, Elliott (1998), "Red men, princess Pocahontas, and George Washington: Harmonizing race relations in Laredo at the turn of the century", The Western Historical Quarterly, 29 (1), Utah State University-The Western History Association, Logan-Fairbanks, pp. $48-85$.

Zúniga, Víctor (1998), “Representaciones infantiles de la frontera y del espacio nacional (materiales para una geografía cultural de la frontera México-Estados Unidos)", en Víctor Zúniiga (coord.), Voces de la frontera. Estudios sobre la dispersión cultural en la frontera México-Estados Unidos, Universidad Autónoma de Nuevo León, Monterrey, pp. 221-300.

Recibido: 17 de diciembre de 2013. Reenviado: 15 de agosto de 2014. Aceptado: 6 de octubre de 2014.

Xavier Oliveras-González. Es doctor en geografía por la Universitat Autònoma de Barcelona, España. Actualmente es investigador del Departamento de Estudios Urbanos y Medio Ambiente en El Colegio de la Frontera Norte, en la sede regional de Matamoros, Tamaulipas. Su línea de investigación actual es la construcción de regiones transfronterizas. Entre sus últimas publicaciones destacan: "La cooperación transfronteriza en la Cerdanya (frontera España-Francia)", Boletín de la Asociación de Geógrafos Españoles, 62, Asociación de Geógrafos Españoles, Madrid, pp. 25-48 (2013); en coautoría, "A typology of agents and subjects of regional cooperation: the experience of the Mediterranean Arch", en N. Bellini y U. Hilpert (eds.), Europe's Changing Geography. The Impact of Inter-regional Networks, Routledge-Regional Studies Association, Londres, pp. 101-123 (2013); "La cooperació transfronterera en la gestió de conques fluvials a la frontera franco-andorrano-espanyola", Estudis d'Història Agrària, 24, Universitat de Barcelona, Barcelona, pp. 233-249 (2012). 